\title{
Studio epidemiologico descrittivo sulle lesioni cutanee croniche nelle Cure Domiciliari dei distretti di Sassari - Alghero - Ozieri
}

\author{
Laura Dettori \\ Infermiera delle Cure Domiciliari del distretto di Sassari, Italia
}

\begin{abstract}
RIASSUNTO
Dall'analisi della letteratura attuale emerge il fatto che non ci siano studi epidemiologici che coinvolgano pazienti con lesioni cutanee croniche nella regione Sardegna. Si è voluto perciò analizzare ed elaborare dei dati a tal proposito per ottenere dei valori sul numero dei pazienti con lesioni cutanee nelle Cure Domiciliari della Provincia di Sassari ma anche per capire quali fossero le problematiche dei vari professionisti sanitari nel gestire tale tipologia di paziente. Per la rilevazione dei dati è stato utilizzato uno strumento unico somministrato agli infermieri del territorio, dove è emersa un'alta percentuale di pazienti con lesioni cutanee croniche e si è riscontrata la necessità di uno specialista in Wound Care. Per la prima volta sono stati ottenuti dati numericamente rilevanti per la regione Sardegna.
\end{abstract}

\section{INTRODUZIONE}

Nell'ulcera la cicatrizzazione è alterata da un processo infiammatorio persistente autoalimentato, il cui quadro istologico è contraddistinto da un'eccessiva proliferazione dei capillari con fibroblasti, linfociti e macrofagi. ${ }^{1,2}$ L'ulcera cutanea non deve essere considerata come malattia a sé, ma come manifestazione secondaria o accessoria, relativa a un altro processo morboso di cui è nel contempo sintomo e segno.

Un'accurata anamnesi, un attento esame obiettivo dell'ulcera e del paziente, unitamente a indagini diagnostiche ed ematochimiche, porteranno a scoprire la patogenesi della lesione $\mathrm{e}$ a intraprendere il più corretto trattamento sistemico e locale di quest'ultima. ${ }^{3}$

L'entità del fenomeno è importante, sia per il numero

Corrispondenza: Laura Dettori, Infermiera delle Cure Domiciliari del distretto di Sassari, Italia.

Tel.: 3491415541.

E-mail: lauradettori4@libero.it

Parole chiave: studio epidemiologico, lesioni cutanee croniche, cure domiciliari, Sardegna.

Conflitto di interesse: l'autrice dichiara l'assenza di conflitti di interesse.

Ricevuto per la pubblicazione: 22 gennaio 2020 .

Accettato per la pubblicazione: 31 ottobre 2020

This work is licensed under a Creative Commons Attribution NonCommercial 4.0 License (CC BY-NC 4.0).

${ }^{\circ}$ Copyright: the Author(s), 2020

Licensee PAGEPress, Italy

Italian Journal of Wound Care 2020; 4(2):53-60

doi:10.4081/ijwc.2020.57 di pazienti coinvolti che per i tempi e le risorse necessari per il trattamento del problema.

Rappresenta una patologia ad elevato significato sociale sia in termini di spesa pubblica e assistenziale che di perdita di giornate lavorative. Nella pratica clinica esse sono di consueto riscontro, nonostante ciò, l'estensione del problema è relativamente poco conosciuta per la mancanza di studi epidemiologici su vasta scala. ${ }^{4}$

Per quanto riguarda il panorama italiano, importanza è data dal progetto S.I.U.C (Studio Italiano Ulcere Cutanee) del 2015-1016, ${ }^{5}$ dove però non vi sono i dati report conclusivi per la regione Sardegna.

Vista l'assenza di dati, è nata l'idea di sviluppare questo lavoro di studio con l'esigenza di approfondire il quadro epidemiologico di una vasta area del Nord Sardegna. È stato scelto di utilizzare uno studio epidemiologico descrittivo, e gli obiettivi sono principalmente: i) di monitorare il fenomeno delle lesioni cutanee croniche nelle Cure Domiciliari nei Distretti di Sassari, Alghero e Ozieri; ii) ottenere una distribuzione delle lesioni cutanee per eziologia del territorio preso in esame; iii) comprendere la gestione infermieristica del paziente in Assistenza Domiciliare Integrata (ADI) con lesioni cutanee croniche; iv) capire l'opinione degli operatori sulla figura dell'infermiere specialista in Wound Care.

\section{MATERIALI E METODI}

Per questo lavoro si è deciso di utilizzare uno studio epidemiologico descrittivo. Tale studio ha previsto la rilevazione diretta dei dati, tramite dei questionari somministrati agli infermieri del territorio. Ai fini dello studio sono stati osservati tutti i pazienti inseriti nelle Cure Domiciliari Integrate dei Distretti di Sassari, Alghero e Ozieri nel periodo predefinito e selezionati in base ai criteri d'in- 
clusione, pazienti con un'attivata assistenza domiciliare integrata ed esclusione i pazienti con attivata solo cure Prestazionali.

Il periodo di effettuazione dello studio è stato individuato dal 1 aprile 2019 al 1 maggio 2019. È stato scelto di rilevare i dati in un periodo di un mese per far si che gli operatori avessero il tempo di vedere tutti i pazienti con attiva assistenza domiciliare integrata (alcuni pazienti hanno previsti uno o due accessi mensili).

Per la rilevazione dei dati è stato utilizzato in tutto il territorio progetto di studio, uno strumento unico elaborato per questo lavoro.

Lo strumento è formato da due parti, la parte 1 relativa al paziente, e la parte 2 relativa all'operatore che gestisce i pazienti in ADI.

La parte 1 è composta da 12 domande. Vi sono tre domande poste ad individuare la fascia di età del paziente, comorbilità e assunzione o meno di terapia anticoagulante. Le domande successive richiamano l'attenzione su dati specifici quali presenza di lesioni cutanee nel paziente ma anche numero, tipologia e durata della lesione; utilizzo o meno di scale di valutazione, utilizzo di medicazioni semplici o avanzate, descrizione dello schema di medicazione e prodotti utilizzati e richiesta di consulenza chirurgica o di uno specialista durante il trattamento.

L'ultima parte comprende l'utilizzo della Scala di Braden come scala di valutazione del rischio.

La parte 2 è composta da 3 domande. La prima che vuole comprendere la conoscenza degli operatori sulla figura dell'infermiere specialista in Wound Care, la seconda indaga sull'opinione degli operatori sull'utilità di uno specialista Wound Care nel territorio e la terza che vuole mettere alla luce le problematiche che incontrano gli operatori durante la gestione di un paziente con lesioni cutanee croniche.

L'inserimento dei dati è stato eseguito con il software Microsoft Access e l'elaborazione degli stessi è avvenuta con Microsoft Excel e Microsoft Access. Ai fini del rispetto della normativa sulla privacy i dati non contengono dati sensibili.

\section{RISULTATI}

Come riferito dai vari coordinatori dei distretti, il numero complessivo dei pazienti in ADI nel mese di Aprile 2019 era 1074, 718 pazienti nel distretto di Sassari, 256 nel distretto di Alghero e 100 nel Distretto di Ozieri.

I questionari compilati relativi al paziente sono stati 654 .

È risultato che il 10,24\% dei pazienti hanno età inferiore a 60 anni (n=67 pz); il 37,57\% hanno tra i 60 e 80 anni $(\mathrm{n}=213)$ e il $57,19 \%$ hanno età superiore a 80 anni $(\mathrm{n}=374)$. In relazione alla presenza di comorbilità si è osservato che il $22,02 \%$ di pazienti presenta diabete $(\mathrm{n}=144)$; il $42,05 \%$ presenta patologie cardiovascolari $(\mathrm{n}=275)$ e il $58,72 \%$ presenta sindrome da allettamento $(\mathrm{n}=384)$. Un ulteriore dato emerso è che il $44,19 \%$ della popolazione di studio assume farmaci anticoagulanti $(\mathrm{n}=289)$.

È stato rilevato che il 50,46\% dei pazienti $(n=330)$ ha presentato lesioni cutanee al momento dello studio (Figura 1) con presenza di più di una lesione nel $29,20 \%$ dei casi, con una media perciò di 1,20 lesioni per paziente.

Sono state quantificate tutte le lesioni presenti nel campione di studio, ottenendo un totale di numero di lesioni di $785(330+455)$.

In relazione alla genesi della lesione, si osserva che nel $77,27 \%$ dei pazienti vi sono lesioni da pressione $(\mathrm{n}=255)$, nel $13,94 \%$ sono presenti ulcere vascolari $(\mathrm{n}=46)$, nel $4,55 \%$ skin tears $(\mathrm{n}=15)$, nel $3,64 \%$ è presente piede diabetico $(\mathrm{n}=12)$, e nel $3,03 \%$ rispettivamente presenti lesione tumorale e ulcera atipica e nel 2,42\% l'infermiere non riesce ad identificare la lesione $(n=8)$.

Di seguito nella Figura 2 si descrive la distribuzione delle lesioni cutanee per eziologia:

È risultato che su 330 lesioni cutanee croniche il $43,0 \%$ è presente da più di 6 mesi ( $\mathrm{n}=142)$, il $28,2 \%$ è presente da $3-10$ settimane ( $\mathrm{n}=93$ ), il $23,9 \%$ da più di 3 mesi $(n=79)$ e il $4,8 \%$ da meno di una settimana $(n=16)$.

Dall'elaborazione dei dati si evince che nel 90,21\% $(\mathrm{n}=590)$ non si utilizzano scale di valutazione nel corso della gestione delle lesioni (Figura 3).

Dallo studio è emerso che nel 34,35\% vengono utilizzate medicazioni semplici $(\mathrm{n}=224)$, nel 18,50\% vengono utilizzate medicazioni avanzate $(\mathrm{n}=121)$ e nel $4,2 \%$ vengono utilizzate entrambe $(\mathrm{n}=14)$.

Attraverso una complicata rielaborazione dei dati è stato possibile elencare e suddividere tutte le medicazioni utilizzate dagli infermieri (Figura 4).

Dalla rielaborazione dei dati si è potuto constatare che la detersione con $\mathrm{NaCl} 0,9 \%$ viene utilizzata nel $80,61 \%$

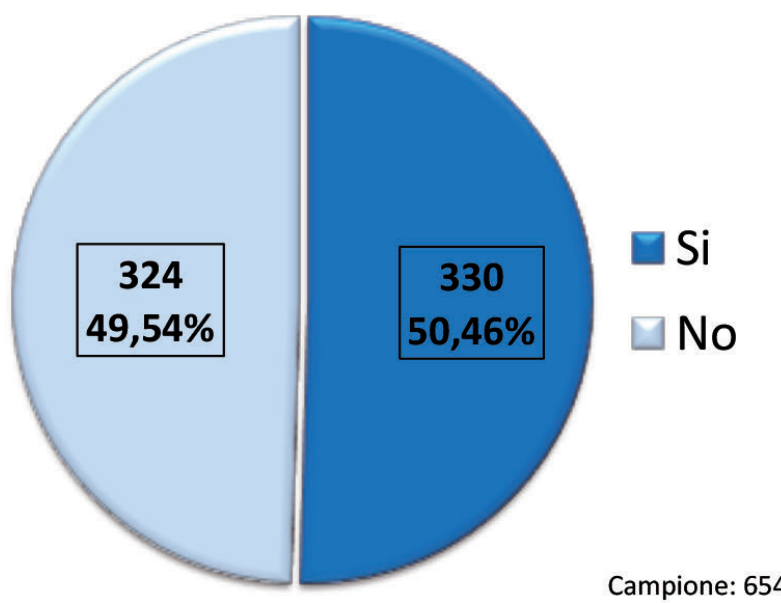

Figura 1. Percentuale pazienti con lesioni cutanee croniche. 
delle lesioni ( $\mathrm{n}=266)$, e solo 1'1,21\% viene detersa con acqua e sapone $(n=4)$. L'utilizzo di Prontosan soluzione per la detersione è del $25,76 \%(\mathrm{n}=85)$.

La disinfezione con Iodopovidone soluzione alcolica viene utilizzata nel 26,06\% $(n=86)$, l'utilizzo di Amukine Med $0,05 \%$ è del 3,94\% $(n=13)$, di Eosina $2 \%$ soluzione cutanea è del $1,82 \%(n=6)$, di perossido di idrogeno $(\mathrm{H} 2 \mathrm{O} 2)$ è dello $0,91 \%(\mathrm{n}=3)$, di Euclorina $2,5 \%$ polvere solubile è dello $0,30 \%(n=1)$ e di merbromina (noto Mercurocromo) è del $0,30 \%(n=1)$.

Per le medicazioni bioattive si ha un utilizzo di Connettivina garza nel 31,52\% delle lesioni ( $n=104)$, di Connettivina crema nel 30,30\% $(n=100)$, di Vulnamin gel nel $23,33 \%(n=77)$, di garza grassa nel 3,03\% $(n=10)$, di Vulnamin crema nel $2,12 \%(n=7)$ e di dermotrofina crema nello $0,91 \%(n=3)$.

Per quanto riguarda l'utilizzo di medicazioni enzimatiche, viene utilizzata la collagenasi nel 44,55\% delle lesioni $(n=147)$.

L'utilizzo della schiuma di poliuretano è dell' $11,82 \%$ $(n=39)$, dell'idrofibra del 10,30\% $(n=34)$, dell'alginato del $8,79 \%(n=29)$, dell'idrogel è del 5,15\% $(n=17)$, e dell'idrocolloide del 0,61\% (2).

L'utilizzo di medicazione antisettiche è presentato dal Sofargen crema con il 10,91\% $(n=36)$, dall' idrofibra Ag+ con il 10,00\% $(n=33)$, dall'Alginato $\mathrm{Ag}+$ con il 3,33\% $(\mathrm{n}=11)$, dalla schiuma di poliuretano $\mathrm{Ag}+$ con lo $0,91 \%$ $(n=3)$, da medicazioni al miele con lo $0,61 \%(n=2)$ e da medicazioni al carbone con lo $0,61 \%(n=2)$.

Altri prodotti come film di poliuretano rappresentano il $9,39 \%(n=31)$, spray protettivi rappresentano il $2,12 \%$ $(\mathrm{n}=7)$, Rigenoma crema lo $0,30 \%(\mathrm{n}=1)$, Gentamicina pomata lo $0,30 \%(n=1)$, cortisonici topici lo $0,61 \%(n=2)$, utilizzo di Tranex fiale lo $0,30 \%(n=1)$ e idrobenda lo $0,30 \%(n=1)$. L'utilizzo del bendaggio elastocompressivo invece rappresenta il 3,03\% $(n=10)$.

Nel campione oggetto di studio, nel $41,59 \%$ è stato richiesta consulenza chirurgica o di uno specialista $(n=272)$.

Infine, dalla compilazione della Scala di Braden è emerso che il $26,15 \%(n=171)$ è risultato paziente ad alto rischio di insorgenza di lesione, nel 40,21\% ( $n=263)$ paziente a rischio e nel $33,64 \%(n=220)$ paziente non a rischio di insorgenza.

La Figura 5 riassume i dati del questionario Parte 1PAZIENTE.

Vi è stata una seconda elaborazione dei dati prendendo in considerazione solo il numero dei pazienti con lesioni cutanee $(n=330)$.

Su 330 pazienti con lesioni cutanee il 73,64\% $(n=243)$ ha richiesto consulenza chirurgica e il 26,36\% no $(n=87)$.

Dato significativo è stato quello della percentuale di lesioni multiple sul singolo paziente; sono stati presi in considerazione i pazienti con una tipologia di lesione e si

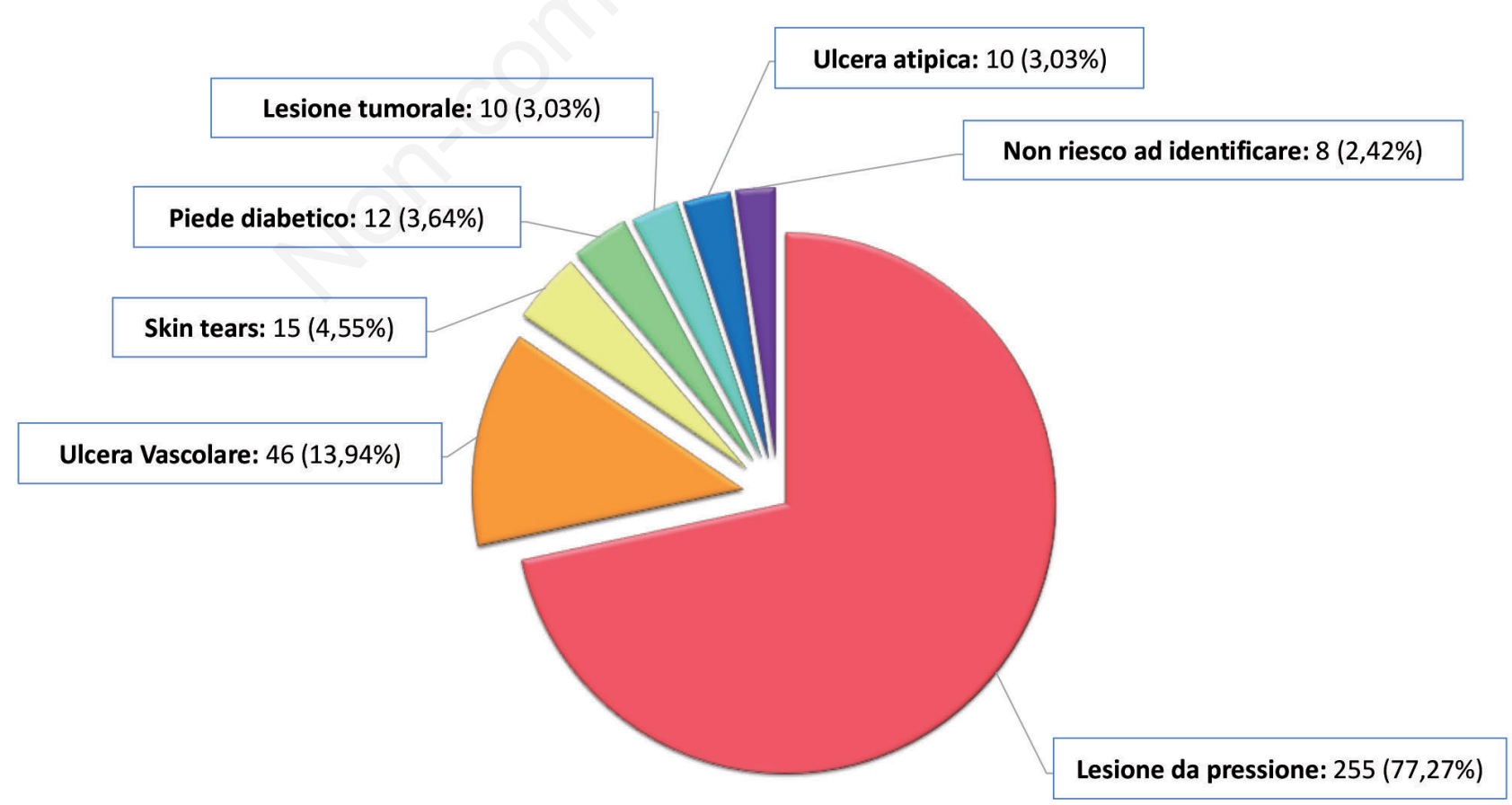

Figura 2. Distribuzione delle ulcere cutanee per eziologia. 
è valutato se fossero presenti anche lesioni di altra eziologia. Nei pazienti con lesioni da pressione $(n=255)$ il $3,53 \%$ ha presentato anche ulcere vascolari $(n=9)$, il $3,14 \%$ ha presentato anche skin tears $(\mathrm{n}=8), 1^{\prime} 1,57 \%$ ha presentato anche piede diabetico, lo $0,78 \%$ ha presentato anche lesioni dove l'infermiere non è riuscito ad identificare l'eziologia $(\mathrm{n}=2)$, e lo $0,39 \%$ ha presentato un'ulcera atipica $(\mathrm{n}=1)$.

Nei pazienti con ulcere vascolari $(n=46)$ il $19,57 \%$ ha presentato anche lesioni da pressione $(\mathrm{n}=9)$, il $6,52 \%$ ha presentato anche piede diabetico $(n=3)$ e il $2,17 \%$ ha presentato skin tears $(\mathrm{n}=1)$.

Nei pazienti con skin tears $(\mathrm{n}=15)$ il $53,33 \%$ ha presentato lesioni da pressione $(\mathrm{n}=8)$ e il $6,67 \%$ ha presentato ulcere vascolari $(\mathrm{n}=1)$.

Nei pazienti con piede diabetico $(n=12)$ il $33,33 \%$ ha presentato lesioni da pressione ( $\mathrm{n}=4$ ) il $25,00 \%$ ha presentato ulcere vascolari $(\mathrm{n}=3)$.

Nei pazienti con ulcera atipica $(n=10)$ il $10,00 \%$ ha presentato anche lesioni da pressione $(\mathrm{n}=1)$.

Nei pazienti in cui l'infermiere non è riuscito ad identificare la lesione $(\mathrm{n}=8)$ il $25,00 \%$ ha presentato anche lesioni da pressione $(\mathrm{n}=2)$.

Nei pazienti con lesione tumorale $(\mathrm{n}=10)$ non si è presentato nessun altro tipo di lesione in concomitanza al momento della raccolta dei dati.

La Figura 6 riassume i dati dei pazienti con lesioni cutanee che mostrano richiesta di visita chirurgica o specialistica e percentuale di lesioni multiple sul singolo paziente.

Prendendo in considerazione solo i pazienti con lesioni $(n=330)$ si è analizzato inoltre che 139 pazienti presentavano solo una lesione nel momento dello studio e invece 191 pazienti presentavano più di una lesione. Gli

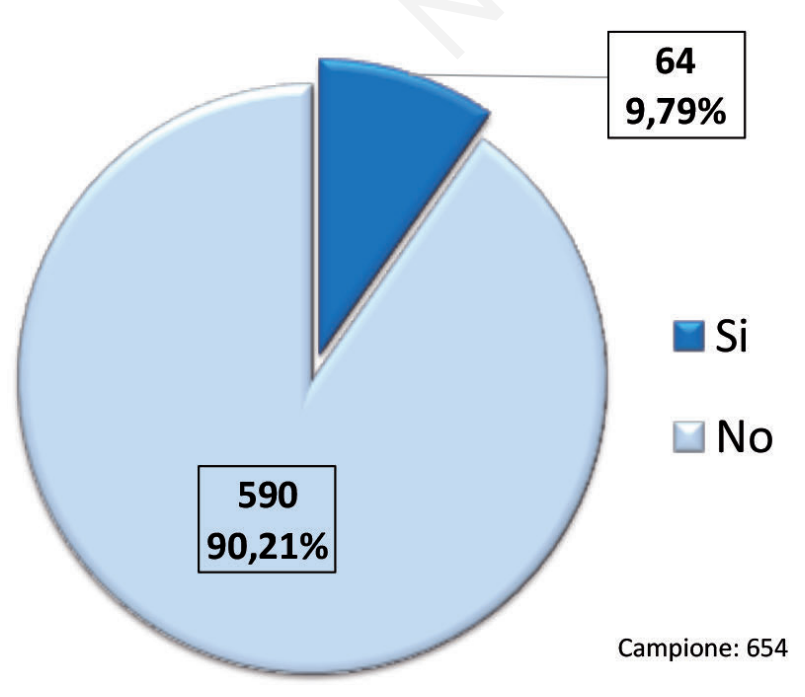

Figura 3. Percentuale utilizzo scala di valutazione. infermieri hanno selezionato il numero esatto di lesioni presenti e si è riuscito ad elaborare il numero di lesioni totali, ovvero 785. Con una media perciò di 2,38 lesioni per paziente (con lesione). Come già detto precedentemente però la media delle lesioni per paziente di tutta la popolazione soggetta di studio è 1,20.

$\mathrm{Si}$ è analizzata inoltre la correlazione tra età e pazienti con lesioni. Su 330 pazienti con lesione cutanea il $63,3 \%$ ha più di 80 anni $(n=210)$, il $28,5 \%$ ha tra i 60 e 80 anni $(\mathrm{n}=94)$ e il 7,9\% ha meno di 60 anni $(\mathrm{n}=26)$. Su $191 \mathrm{pa}-$ zienti che hanno presentato più di una lesione invece il $61,8 \%$ ha più di 80 anni $(n=118)$, il $30,4 \%$ ha tra i 60 e 80 anni $(n=58)$ e il $7,9 \%$ ha meno di 60 anni $(n=15)$.

Si è studiata la relazione tra pazienti con lesioni e le patologie (diabete, patologie cardiovascolari e sindrome da allettamento). Su 330 pazienti con lesioni 213 presentano sindrome da allettamento, 160 presentano patologie cardiovascolari e 85 presentano diabete.

$\mathrm{Da}$ tener presente che avere una di queste patologie non esclude non avere l'altra; infatti su 213 pazienti con sindrome da allettamento 89 presentano anche patologie cardiovascolari e 46 invece presentano anche diabete.

Cosi per 160 pazienti con patologie cardiovascolari, 89 presentano sindrome da allettamento e 53 presentano anche diabete.

Su 85 pazienti con diabete 46 presentano sindrome da allettamento e 53 presentano anche patologie cardiovascolari.

Per la raccolta dati relativa all'operatore sono stati considerati 58 questionari, con un'adesione del 77.33\%. I questionari sono stati distribuiti a 75 infermieri del territorio: 43 infermieri del distretto di Sassari, 21 di Alghero e 10 di Ozieri.

Il dato più significativo emerso è che il 98,28\% degli operatori $(n=57)$ sostiene di ritenere utile la presenza di uno specialista Wound Care in grado di coordinare i vari professionisti ed impostare adeguate strategie terapeutiche.

I dati complessivi del questionario operatore sono riportati nella Figura 7.

\section{DISCUSSIONE}

I dati inseriti risentono della compilazione da parte di più operatori e della differenza di gestione di questi ultimi, pertanto l'analisi dovrà sempre tenere ben presente la metodologia adottata. Il 57,19\% $(\mathrm{n}=374)$ dei pazienti in Assistenza Domiciliare Integrata dei distretti di Sassari, Alghero e Ozieri risulta essere in una fascia d'età di più di 80 anni, con sindrome da allettamento nel $58,72 \%$ dei casi $(\mathrm{n}=384)$.

Ma un dato interessante che emerge è l'alta percentuale, il 50,3\% dei pazienti totali $(\mathrm{n}=330)$ che presenta una lesione cutanea nel momento della rilevazione dei dati.

Un dato significativo è sicuramente dato dalla distribu- 
zione delle lesioni cutanee per eziologia; dove il $72 \%$ è rappresentato dalle lesioni da pressione, il $13 \%$ da ulcere vascolari e la restante parte, il $4 \%$ da skin tears, il 3\% rispettivamente da piede diabetico, lesione tumorale e ulcera atipica e il $2 \%$ dove l'infermiere non riesce ad identificare il tipo di lesione. Nonostante sia emersa un'alta percentuale rappresentata dalle lesioni di pressione, se paragonato ai risultati dello Studio Italiano Ulcere Cutanee (2015-2016) nel setting assistenziale ADI, in cui si afferma che oltre il 95\% dei pazienti allettati o non autosufficienti sono affetti da lesioni da pressione, ${ }^{5}$ la stima in questo campione di studio risulta essere più bassa.

Importanza è data anche dal tempo di durata delle lesioni cutanee poiché i dati che emergono mostrano che il $43,0 \%$ delle lesioni $(\mathrm{n}=142)$ è presente da più di 6 mesi, mettendo alla luce la problematica della cronicità delle lesioni ma anche sulla difficoltà di risoluzione di esse.

Dalla rielaborazione dei dati si è riusciti a studiare non solo il numero di pazienti con lesioni cutanee $(n=330)$ ma anche il numero complessivo ed effettivo di tutte le lesioni

\begin{tabular}{|c|c|c|c|c|c|c|c|c|c|c|c|c|c|c|c|c|c|c|c|c|}
\hline \multirow[b]{2}{*}{ TOTALI } & \multicolumn{2}{|c|}{$\begin{array}{c}\text { Totale } \mathrm{pz} \\
\text { con lesioni }\end{array}$} & \multicolumn{2}{|c|}{$\begin{array}{c}\text { Totale pz } \\
\text { chirurgo SI }\end{array}$} & \multicolumn{2}{|c|}{$\begin{array}{c}\text { Totale pz } \\
\text { chirurgo } \\
\text { NO }\end{array}$} & \multicolumn{2}{|c|}{$\begin{array}{l}\text { Lesione da } \\
\text { pressione }\end{array}$} & \multicolumn{2}{|c|}{$\begin{array}{c}\text { Ulcera } \\
\text { vascolare }\end{array}$} & \multicolumn{2}{|c|}{ Skin tears } & \multicolumn{2}{|c|}{$\begin{array}{c}\text { Piede } \\
\text { diabetico }\end{array}$} & \multicolumn{2}{|c|}{$\begin{array}{l}\text { Lesione } \\
\text { Tumorale }\end{array}$} & \multicolumn{2}{|c|}{$\begin{array}{l}\text { Ulcera } \\
\text { atipica }\end{array}$} & \multicolumn{2}{|c|}{\begin{tabular}{|c} 
Non riesco \\
ad \\
identificare
\end{tabular}} \\
\hline & 330 & $\%$ & 243 & $73,64 \%$ & 87 & $26,36 \%$ & 255 & $77,27 \%$ & 46 & $13,94 \%$ & 15 & $4,55 \%$ & 12 & $3,64 \%$ & 10 & $3,03 \%$ & 10 & $3,03 \%$ & 8 & $2,42 \%$ \\
\hline \multicolumn{21}{|l|}{ MEDICAZIONI UTILIZZATE } \\
\hline $\mathrm{Nacl}$ & 266 & $80,61 \%$ & 190 & $78,19 \%$ & 76 & $87,36 \%$ & 193 & $75,69 \%$ & 36 & $78,26 \%$ & 8 & $53,33 \%$ & 8 & $66,67 \%$ & 9 & $90,00 \%$ & 6 & $60,00 \%$ & 6 & $75,00 \%$ \\
\hline lodopor & 86 & $26,06 \%$ & 63 & $25,93 \%$ & 23 & $26,44 \%$ & 54 & $21,18 \%$ & 18 & $39,13 \%$ & 3 & $20,00 \%$ & 3 & $25,00 \%$ & 3 & $30,00 \%$ & 2 & $20,00 \%$ & 3 & $37,50 \%$ \\
\hline $\mathrm{H} 2 \mathrm{O2}$ & 3 & $0,91 \%$ & 2 & $0,82 \%$ & 1 & $1,15 \%$ & 2 & $0,78 \%$ & 0 & $0,00 \%$ & 0 & $0,00 \%$ & 0 & $0,00 \%$ & 1 & $10,00 \%$ & 0 & $0,00 \%$ & 0 & $0,00 \%$ \\
\hline Prontosan & 85 & $25,76 \%$ & 84 & $34,57 \%$ & 1 & $1,15 \%$ & 65 & $25,49 \%$ & 7 & $15,22 \%$ & 4 & $26,67 \%$ & 5 & $41,67 \%$ & 2 & $20,00 \%$ & 2 & $20,00 \%$ & 0 & $0,00 \%$ \\
\hline Amukine Med & 13 & $3,94 \%$ & 13 & $5,35 \%$ & 0 & $0,00 \%$ & 7 & $2,75 \%$ & 2 & $4,35 \%$ & 0 & $0,00 \%$ & 2 & $16,67 \%$ & 1 & $10,00 \%$ & 1 & $10,00 \%$ & 0 & $0,00 \%$ \\
\hline Acqua es & 4 & $1,21 \%$ & 4 & $1,65 \%$ & 0 & $0,00 \%$ & 1 & $0,39 \%$ & 2 & $4,35 \%$ & 1 & $6,67 \%$ & 0 & $0,00 \%$ & 0 & $0,00 \%$ & 0 & $0,00 \%$ & 0 & $0,00 \%$ \\
\hline Euclorina & 1 & $0,30 \%$ & 1 & $0,41 \%$ & 0 & $0,00 \%$ & 0 & $0,00 \%$ & 1 & $2,17 \%$ & 0 & $0,00 \%$ & 0 & $0,00 \%$ & 0 & $0,00 \%$ & 0 & $0,00 \%$ & 0 & $0,00 \%$ \\
\hline Eosina & 6 & $1,82 \%$ & 6 & $2,47 \%$ & 0 & $0,00 \%$ & 2 & $0,78 \%$ & 1 & $2,17 \%$ & 2 & $13,33 \%$ & 0 & $0,00 \%$ & 0 & $0,00 \%$ & 0 & $0,00 \%$ & 1 & $12,50 \%$ \\
\hline Mercurio cromo & 1 & $0,30 \%$ & 1 & $0,41 \%$ & 0 & $0,00 \%$ & 1 & $0,39 \%$ & 0 & $0,00 \%$ & 0 & $0,00 \%$ & 0 & $0,00 \%$ & 0 & $0,00 \%$ & 0 & $0,00 \%$ & 0 & $0,00 \%$ \\
\hline Connettivina c & 100 & $30,30 \%$ & 61 & $25,10 \%$ & 39 & $44,83 \%$ & 74 & $29,02 \%$ & 8 & $17,39 \%$ & 8 & $53,33 \%$ & 0 & $0,00 \%$ & 3 & $30,00 \%$ & 4 & $40,00 \%$ & 3 & $37,50 \%$ \\
\hline Connettivina & 104 & $31,52 \%$ & 73 & $30,04 \%$ & 31 & $35,63 \%$ & 73 & $28,63 \%$ & 19 & $41,30 \%$ & 3 & $20,00 \%$ & 0 & $0,00 \%$ & 3 & $30,00 \%$ & 5 & $50,00 \%$ & 1 & $12,50 \%$ \\
\hline Collag & 147 & $44,55 \%$ & 124 & $51,03 \%$ & 23 & $26,44 \%$ & 114 & $44,71 \%$ & 21 & $45,65 \%$ & 1 & $6,67 \%$ & 4 & $33,33 \%$ & 4 & $40,00 \%$ & 3 & $30,00 \%$ & 0 & $0,00 \%$ \\
\hline Sofargen & 36 & $10,91 \%$ & 15 & $6,17 \%$ & 21 & $24,14 \%$ & 30 & $11,76 \%$ & 1 & $2,17 \%$ & 1 & $6,67 \%$ & 0 & $0,00 \%$ & 0 & $0,00 \%$ & 0 & $0,00 \%$ & 4 & $50,00 \%$ \\
\hline Gentamicina & 1 & $0,30 \%$ & 1 & $0,41 \%$ & 0 & $0,00 \%$ & 0 & $0,00 \%$ & 0 & $0,00 \%$ & 0 & $0,00 \%$ & 0 & $0,00 \%$ & 0 & $0,00 \%$ & 1 & $10,00 \%$ & 0 & $0,00 \%$ \\
\hline Cortisonici & 2 & $0,61 \%$ & 2 & $0,82 \%$ & 0 & $0,00 \%$ & 0 & $0,00 \%$ & 1 & $2,17 \%$ & 0 & $0,00 \%$ & 0 & $0,00 \%$ & 0 & $0,00 \%$ & 1 & $10,00 \%$ & 0 & $0,00 \%$ \\
\hline Vulna & 7 & $2,12 \%$ & 4 & $1,65 \%$ & 3 & $3,45 \%$ & 6 & $2,35 \%$ & 0 & $0,00 \%$ & 0 & $0,00 \%$ & 0 & $0,00 \%$ & 1 & $10,00 \%$ & 0 & $0,00 \%$ & 0 & $0,00 \%$ \\
\hline Vulnamin gel & 77 & $23,33 \%$ & 74 & $30,45 \%$ & 3 & $3,45 \%$ & 57 & $22,35 \%$ & 11 & $23,91 \%$ & 1 & $6,67 \%$ & 7 & $58,33 \%$ & 1 & $10,00 \%$ & 0 & $0,00 \%$ & 0 & $0,00 \%$ \\
\hline Idrogel & 17 & $5,15 \%$ & 16 & $6,58 \%$ & 1 & $1,15 \%$ & 15 & $5,88 \%$ & 0 & $0,00 \%$ & 1 & $6,67 \%$ & 1 & $8,33 \%$ & 0 & $0,00 \%$ & 0 & $0,00 \%$ & 0 & $0,00 \%$ \\
\hline Rigenoma crema & 1 & $0,30 \%$ & 1 & $0,41 \%$ & 0 & $0,00 \%$ & 0 & $0,00 \%$ & 1 & $2,17 \%$ & 0 & $0,00 \%$ & 0 & $0,00 \%$ & 0 & $0,00 \%$ & 0 & $0,00 \%$ & 0 & $0,00 \%$ \\
\hline Medicazione al $\mathrm{r}$ & 2 & $0,61 \%$ & 2 & $0,82 \%$ & 0 & $0,00 \%$ & 2 & $0,78 \%$ & 0 & $0,00 \%$ & 0 & $0,00 \%$ & 0 & $0,00 \%$ & 0 & $0,00 \%$ & 0 & $0,00 \%$ & 0 & $0,00 \%$ \\
\hline Dermotrofina & 3 & $0,91 \%$ & 2 & $0,82 \%$ & 1 & $1,15 \%$ & 2 & $0,78 \%$ & 0 & $0,00 \%$ & 1 & $6,67 \%$ & 0 & $0,00 \%$ & 0 & $0,00 \%$ & 0 & $0,00 \%$ & 0 & $0,00 \%$ \\
\hline Spray & 7 & $2,12 \%$ & 5 & $2,06 \%$ & 2 & $2,30 \%$ & 6 & $2,35 \%$ & 0 & $0,00 \%$ & 1 & $6,67 \%$ & 0 & $0,00 \%$ & 0 & $0,00 \%$ & 0 & $0,00 \%$ & 0 & $0,00 \%$ \\
\hline Tranex & 1 & $0,30 \%$ & 1 & $0,41 \%$ & 0 & $0,00 \%$ & 0 & $0,00 \%$ & 0 & $0,00 \%$ & 0 & $0,00 \%$ & 0 & $0,00 \%$ & 1 & $10,00 \%$ & 0 & $0,00 \%$ & 0 & $0,00 \%$ \\
\hline Garza gr & 10 & $3,03 \%$ & 8 & $3,29 \%$ & 2 & $2,30 \%$ & 6 & $2,35 \%$ & 2 & $4,35 \%$ & 1 & $6,67 \%$ & 0 & $0,00 \%$ & 1 & $10,00 \%$ & 0 & $0,00 \%$ & 0 & $0,00 \%$ \\
\hline Medicazione al carbone & 2 & $0,61 \%$ & 2 & $0,82 \%$ & 0 & $0,00 \%$ & 1 & $0,39 \%$ & 1 & $2,17 \%$ & 0 & $0,00 \%$ & 0 & $0,00 \%$ & 0 & $0,00 \%$ & 0 & $0,00 \%$ & 0 & $0,00 \%$ \\
\hline Idrocoll & 2 & $0,61 \%$ & 2 & $0,82 \%$ & 0 & $0,00 \%$ & 1 & $0,39 \%$ & 0 & $0,00 \%$ & 0 & $0,00 \%$ & 1 & $8,33 \%$ & 0 & $0,00 \%$ & 0 & $0,00 \%$ & 0 & $0,00 \%$ \\
\hline Idrofibra & 34 & $10,30 \%$ & 34 & $13,99 \%$ & 0 & $0,00 \%$ & 26 & $10,20 \%$ & 4 & $8,70 \%$ & 1 & $6,67 \%$ & 2 & $16,67 \%$ & 1 & $10,00 \%$ & 0 & $0,00 \%$ & 0 & $0,00 \%$ \\
\hline Alginato & 29 & $8,79 \%$ & 28 & $11,52 \%$ & 1 & $1,15 \%$ & 26 & $10,20 \%$ & 2 & $4,35 \%$ & 0 & $0,00 \%$ & 1 & $8,33 \%$ & 0 & $0,00 \%$ & 0 & $0,00 \%$ & 0 & $0,00 \%$ \\
\hline Idrofibra AG & 33 & $10,00 \%$ & 33 & $13,58 \%$ & 0 & $0,00 \%$ & 24 & $9,41 \%$ & 4 & $8,70 \%$ & 0 & $0,00 \%$ & 5 & $41,67 \%$ & 0 & $0,00 \%$ & 0 & $0,00 \%$ & 0 & $0,00 \%$ \\
\hline Alginato AG & 11 & $3,33 \%$ & 11 & $4,53 \%$ & 0 & $0,00 \%$ & 7 & $2,75 \%$ & 0 & $0,00 \%$ & 0 & $0,00 \%$ & 2 & $16,67 \%$ & 1 & $10,00 \%$ & 1 & $10,00 \%$ & 0 & $0,00 \%$ \\
\hline Schiuma di poliuretano & 39 & $11,82 \%$ & 39 & $16,05 \%$ & 0 & $0,00 \%$ & 34 & $13,33 \%$ & 2 & $4,35 \%$ & 1 & $6,67 \%$ & 0 & $0,00 \%$ & 1 & $10,00 \%$ & 1 & $10,00 \%$ & 0 & $0,00 \%$ \\
\hline Schiuma di poliuretano AG & 3 & $0,91 \%$ & 3 & $1,23 \%$ & 0 & $0,00 \%$ & 2 & $0,78 \%$ & 1 & $2,17 \%$ & 0 & $0,00 \%$ & 0 & $0,00 \%$ & 0 & $0,00 \%$ & 0 & $0,00 \%$ & 0 & $0,00 \%$ \\
\hline Hydrofilm & 31 & $9,39 \%$ & 30 & $12,35 \%$ & 1 & $1,15 \%$ & 28 & $10,98 \%$ & 0 & $0,00 \%$ & 1 & $6,67 \%$ & 1 & $8,33 \%$ & 0 & $0,00 \%$ & 1 & $10,00 \%$ & 0 & $0,00 \%$ \\
\hline Idrobenda & 1 & $0,30 \%$ & 0 & $0,00 \%$ & 1 & $1,15 \%$ & 1 & $0,39 \%$ & 0 & $0,00 \%$ & 0 & $0,00 \%$ & 0 & $0,00 \%$ & 0 & $0,00 \%$ & 0 & $0,00 \%$ & 0 & $0,00 \%$ \\
\hline Bendaggio elastocompressivo & 10 & $3,03 \%$ & 10 & $4,12 \%$ & 0 & $0,00 \%$ & 0 & $0,00 \%$ & 10 & $21,74 \%$ & 0 & $0,00 \%$ & 0 & $0,00 \%$ & 0 & $0,00 \%$ & 0 & $0,00 \%$ & 0 & $0,00 \%$ \\
\hline
\end{tabular}

Figura 4. Dati visite specialistiche e medicazioni utilizzate. 


\begin{tabular}{|c|c|c|c|c|c|c|c|c|c|}
\hline \multicolumn{2}{|c|}{ QUESTIONARIO PARTE 1 - PAZIENTE } & \multirow{2}{*}{$\begin{array}{l}\text { TOT } \\
654\end{array}$} & \multirow[t]{2}{*}{$\%$} & \multirow{2}{*}{$\begin{array}{c}\text { SASSARI } \\
443\end{array}$} & \multirow{2}{*}{$\begin{array}{c}\% \\
67,74 \%\end{array}$} & \multirow{2}{*}{\begin{tabular}{c|} 
ALGHERO \\
191 \\
\end{tabular}} & \multirow{2}{*}{$\begin{array}{c}\% \\
29,20 \%\end{array}$} & \multirow{2}{*}{$\begin{array}{c}\text { OZIERI } \\
20\end{array}$} & \multirow{2}{*}{$\begin{array}{c}\% \\
3,06 \%\end{array}$} \\
\hline & Risposta & & & & & & & & \\
\hline Domanda 1 & Meno di 60 anni & 67 & $10,24 \%$ & 45 & $10,16 \%$ & 22 & $11,52 \%$ & $\mathbf{0}$ & $0,00 \%$ \\
\hline \multirow{2}{*}{$\begin{array}{l}\text { Individua la fascia di età del paziente in } \\
\text { carico: }\end{array}$} & Tra i 60 e 80 anni & 213 & $32,57 \%$ & 154 & $34,76 \%$ & 54 & $28,27 \%$ & 5 & $25,00 \%$ \\
\hline & Più di 80 anni & 374 & $57,19 \%$ & 244 & $55,08 \%$ & 115 & $60,21 \%$ & 15 & $75,00 \%$ \\
\hline Domanda 2 & Diabete & 144 & $22,02 \%$ & 106 & $23,93 \%$ & 33 & $17,28 \%$ & 5 & $25,00 \%$ \\
\hline \multirow{2}{*}{$\begin{array}{l}\text { \| paziente presenta una o più di queste } \\
\text { patologie? }\end{array}$} & Patologie cardiovascolari & 275 & $42,05 \%$ & 184 & $41,53 \%$ & 82 & $42,93 \%$ & 9 & $45,00 \%$ \\
\hline & Sindrome di allettamento & 384 & $58,72 \%$ & 257 & $58,01 \%$ & 117 & $61,26 \%$ & 10 & $50,00 \%$ \\
\hline Domanda 3 & Si & 289 & $44,19 \%$ & 191 & $43,12 \%$ & 87 & $45,55 \%$ & 11 & $55,00 \%$ \\
\hline $\begin{array}{l}\text { Il paziente assume farmaci anticoagulanti? } \\
\text { (es. Coumadin) }\end{array}$ & No & 365 & $55,81 \%$ & 252 & $56,88 \%$ & 104 & $54,45 \%$ & 9 & $45,00 \%$ \\
\hline Domanda 4 & Si & 330 & $50,46 \%$ & 210 & $47,40 \%$ & 100 & $52,36 \%$ & 20 & $100,00 \%$ \\
\hline $\begin{array}{l}\text { Sono presenti lesioni cutanee nel paziente } \\
\text { in questo dato momento? }\end{array}$ & No & 324 & $49,54 \%$ & 233 & $52,60 \%$ & 91 & $47,64 \%$ & $\mathbf{0}$ & $0,00 \%$ \\
\hline Domanda 5 & Si & 191 & $29,20 \%$ & 117 & $26,41 \%$ & 59 & $30,89 \%$ & 15 & $75,00 \%$ \\
\hline \multirow{3}{*}{$\begin{array}{l}\text { Se la risposta è si, è presente più di una } \\
\text { lesione? }\end{array}$} & TOT lesioni in più & 455 & & 285 & & 122 & & 48 & \\
\hline & Media & 1,20 & & 1,11 & & 1,16 & & 3,4 & \\
\hline & No & 463 & $70,80 \%$ & 326 & $73,59 \%$ & 132 & $69,11 \%$ & 5 & $25,00 \%$ \\
\hline Domanda 6 & Lesione da pressione & 255 & $38,99 \%$ & 164 & $37,02 \%$ & 74 & $38,74 \%$ & 17 & $85,00 \%$ \\
\hline \multirow{7}{*}{$\begin{array}{l}\text { Che tipo di lesione cutanea è presente nel } \\
\text { paziete? }\end{array}$} & Ulcera Vascolare & 46 & $7,03 \%$ & 28 & $6,32 \%$ & 14 & $7,33 \%$ & 4 & $20,00 \%$ \\
\hline & Skin tears & 15 & $2,29 \%$ & 12 & $2,71 \%$ & 3 & $1,57 \%$ & 0 & $0,00 \%$ \\
\hline & Piede diabetico & 12 & $1,83 \%$ & 8 & $1,81 \%$ & 3 & $1,57 \%$ & 1 & $5,00 \%$ \\
\hline & Lesione tumorale & 10 & $1,53 \%$ & 4 & $0,90 \%$ & 5 & $2,62 \%$ & 1 & $5,00 \%$ \\
\hline & Ulcera atipica & 10 & $1,53 \%$ & 5 & $1,13 \%$ & 5 & $2,62 \%$ & $\mathbf{0}$ & $0,00 \%$ \\
\hline & Non riesco ad identificare & 8 & $1,22 \%$ & 6 & $1,35 \%$ & 2 & $1,05 \%$ & $\mathbf{0}$ & $0,00 \%$ \\
\hline & Totale & 356 & & 227 & & 106 & & 23 & \\
\hline Domanda 7 & Meno di 1 settimana & 16 & $2,45 \%$ & 11 & $2,48 \%$ & 5 & $2,62 \%$ & $\mathbf{0}$ & $0,00 \%$ \\
\hline \multirow{3}{*}{$\begin{array}{c}\text { Da quanto tempo vengono riferite le } \\
\text { lesioni? }\end{array}$} & 3-10 settimane & 93 & $14,22 \%$ & 54 & $12,19 \%$ & 35 & $18,32 \%$ & 4 & $20,00 \%$ \\
\hline & Più di 3 mesi & 79 & $12,08 \%$ & 51 & $11,51 \%$ & 23 & $12,04 \%$ & 5 & $25,00 \%$ \\
\hline & Più di 6 mesi & 142 & $21,71 \%$ & 94 & $21,22 \%$ & 37 & $19,37 \%$ & 11 & $55,00 \%$ \\
\hline Domanda 8 & Si & 64 & $9,79 \%$ & 22 & $4,97 \%$ & 37 & $19,37 \%$ & 5 & $25,00 \%$ \\
\hline $\begin{array}{l}\text { Utilizzi scale di valutazione nel corso della } \\
\text { gestione delle lesioni? }\end{array}$ & No & 590 & $90,21 \%$ & 421 & $95,03 \%$ & 154 & $80,63 \%$ & 15 & $75,00 \%$ \\
\hline Domanda 9 & Medicazioni semplici & 224 & $34,25 \%$ & 136 & $30,70 \%$ & 77 & $40,31 \%$ & 11 & $55,00 \%$ \\
\hline Che tipo di medicazioni utilizzi? & Medicazioni avanzate & 121 & $18,50 \%$ & 81 & $18,28 \%$ & 27 & $14,14 \%$ & 13 & $65,00 \%$ \\
\hline Domanda 10 & & \multirow{2}{*}{\multicolumn{2}{|c|}{ Vedi "Figura 4" }} & & & & & & \\
\hline $\begin{array}{l}\text { Descrivi schema di medicazione e prodotti } \\
\text { utilizzati: }\end{array}$ & & & & & & & & & \\
\hline Domanda 11 & Si & 272 & $41,59 \%$ & 180 & $40,63 \%$ & 75 & $39,27 \%$ & 17 & $85,00 \%$ \\
\hline $\begin{array}{l}\text { è stata richiesta consulenza chirurgica o di } \\
\text { uno specialista? }\end{array}$ & No & 382 & $58,41 \%$ & 263 & $59,37 \%$ & 116 & $60,73 \%$ & 3 & $15,00 \%$ \\
\hline Domanda 12 & $<13$ & 171 & $26,15 \%$ & 126 & $28,44 \%$ & 38 & $19,90 \%$ & 7 & $35,00 \%$ \\
\hline Esamina il paziente e compila la seguente & 13-16 & 263 & $40,21 \%$ & 177 & $39,95 \%$ & 79 & $41,36 \%$ & 7 & $35,00 \%$ \\
\hline Braden): & $17-23$ & 220 & $33,64 \%$ & 140 & $31,60 \%$ & 74 & $38,74 \%$ & 6 & $30,00 \%$ \\
\hline
\end{tabular}

Figura 5. Dati questionario paziente. 
presenti nel momento dello studio; si sono valutate infatti un numero di 785 lesioni cutanee, facendo emergere la reale entità del problema.

La media di lesioni per paziente della popolazione di studio è 1,20. Questo sottolinea come in un'alta percen- tuale di casi sono presenti sullo stesso paziente più lesioni ulcerative.

Dato positivo è che su 330 pazienti con lesioni cutanee, nel 73,6\% dei casi $(n=274)$ è stata richiesta visita chirurgica o di uno specialista per visionare le lesioni e per

\begin{tabular}{|c|c|c|c|c|c|c|c|c|c|c|c|c|c|c|c|c|c|c|c|c|}
\hline \multirow[b]{2}{*}{ TOTALI } & \multicolumn{2}{|c|}{$\begin{array}{c}\text { Totale pz } \\
\text { con lesioni }\end{array}$} & \multicolumn{2}{|c|}{$\mid \begin{array}{c}\text { Totale pz } \\
\text { chirurgo SI }\end{array}$} & \multicolumn{2}{|c|}{$\begin{array}{c}\text { Totale pz } \\
\text { chirurgo } \\
\text { NO }\end{array}$} & \multicolumn{2}{|c|}{$\begin{array}{c}\text { Lesione da } \\
\text { pressione }\end{array}$} & \multicolumn{2}{|c|}{$\begin{array}{c}\text { Ulcera } \\
\text { vascolare }\end{array}$} & \multicolumn{2}{|c|}{ Skin tears } & \multicolumn{2}{|c|}{$\begin{array}{c}\text { Piede } \\
\text { diabetico }\end{array}$} & \multicolumn{2}{|c|}{$\begin{array}{l}\text { Lesione } \\
\text { Tumorale }\end{array}$} & \multicolumn{2}{|c|}{$\begin{array}{l}\text { Ulcera } \\
\text { atipica }\end{array}$} & \multicolumn{2}{|c|}{\begin{tabular}{|c} 
Non riesco \\
ad \\
identificare
\end{tabular}} \\
\hline & 330 & $\%$ & 243 & $73,64 \%$ & 87 & $26,36 \%$ & 255 & $77,27 \%$ & 46 & $13,94 \%$ & 15 & $4,55 \%$ & 12 & $3,64 \%$ & 10 & $3,03 \%$ & 10 & $3,03 \%$ & 8 & $2,42 \%$ \\
\hline TIPI DI LESIONE & & & & & & & \multicolumn{14}{|c|}{ IONI MULTIPLE SUL SINGOLO PAZIENTE } \\
\hline Lesione da pressione & 255 & $77,27 \%$ & 189 & $77,78 \%$ & 66 & $75,86 \%$ & & & 9 & $19,57 \%$ & 8 & $53,33 \%$ & 4 & $33,33 \%$ & 0 & $0,00 \%$ & 1 & $10,00 \%$ & 2 & $25,00 \%$ \\
\hline Ulcera vascolare & 46 & $13,94 \%$ & $39 \mid$ & $16,05 \%$ & 7 & $8,05 \%$ & 9 & $3,53 \%$ & & & 1 & $6,67 \%$ & 3 & $25,00 \%$ & 0 & $0,00 \%$ & 0 & $0,00 \%$ & 0 & $0,00 \%$ \\
\hline Skin tears & 15 & $4,55 \%$ & 9 & $3,70 \%$ & 6 & $6,90 \%$ & 8 & $3,14 \%$ & 1 & $2,17 \%$ & & & 0 & $0,00 \%$ & 0 & $0,00 \%$ & 0 & $0,00 \%$ & 0 & $0,00 \%$ \\
\hline Piede diabetico & 12 & $3,64 \%$ & 12 & $4,94 \%$ & 0 & $0,00 \%$ & 4 & $1,57 \%$ & 3 & $6,52 \%$ & 0 & $0,00 \%$ & & & 0 & $0,00 \%$ & 0 & $0,00 \%$ & 0 & $0,00 \%$ \\
\hline Lesione tumorale & 10 & $3,03 \%$ & 7 & $2,88 \%$ & 3 & $3,45 \%$ & 0 & $0,00 \%$ & 0 & $0,00 \%$ & 0 & $0,00 \%$ & 0 & $0,00 \%$ & & & 0 & $0,00 \%$ & 0 & $0,00 \%$ \\
\hline Ulcera atipica & 10 & $3,03 \%$ & 8 & $3,29 \%$ & 2 & $2,30 \%$ & 1 & $0,39 \%$ & 0 & $0,00 \%$ & 0 & $0,00 \%$ & 0 & $0,00 \%$ & 0 & $0,00 \%$ & & & 0 & $0,00 \%$ \\
\hline Non riesco ad identificare & 8 & $2,42 \%$ & 2 & $0,82 \%$ & 6 & $6,90 \%$ & 2 & $0,78 \%$ & 0 & $0,00 \%$ & 0 & $0,00 \%$ & 0 & $0,00 \%$ & 0 & $0,00 \%$ & 0 & $0,00 \%$ & & \\
\hline
\end{tabular}

Figura 6. Dati lesioni multiple.

\begin{tabular}{|c|c|c|c|c|c|c|c|c|c|c|c|}
\hline \multicolumn{4}{|c|}{ QUESTIONARIO PARTE 2 - OPERATORE } & TOT & $\%$ & SASSARI & $\%$ & ALGHERO & $\%$ & OZIERI & $\%$ \\
\hline \multicolumn{4}{|c|}{ TOT QUESTIONARI COMPILATI } & 58 & & 32 & $55,17 \%$ & 21 & $36,21 \%$ & 5 & $8,62 \%$ \\
\hline \multicolumn{3}{|c|}{ Domanda 1} & $\mathrm{Si}$ & 46 & $79,31 \%$ & 23 & $71,88 \%$ & 19 & $90,48 \%$ & 4 & $80,00 \%$ \\
\hline \multicolumn{3}{|c|}{$\begin{array}{l}\text { Hai mai sentito parlare della figura dell'infermiere } \\
\text { specialista i Wound Care? }\end{array}$} & No & 12 & $20,69 \%$ & 9 & $28,13 \%$ & 2 & $9,52 \%$ & 1 & $20,00 \%$ \\
\hline \multicolumn{3}{|c|}{ Domanda 2} & $\mathrm{Si}$ & 57 & $98,28 \%$ & 32 & $100,00 \%$ & 20 & $95,24 \%$ & 5 & $100,00 \%$ \\
\hline \multicolumn{3}{|c|}{$\begin{array}{l}\text { Ritieni utile la presenza di uno specialista Wound } \\
\text { Care in grado di coordinare i vari professionisti ed } \\
\text { impostare adeguate strategie terapeutiche? }\end{array}$} & No & 1 & $1,72 \%$ & $\mathbf{0}$ & $0,00 \%$ & 1 & $4,76 \%$ & $\mathbf{0}$ & $0,00 \%$ \\
\hline \multirow{7}{*}{ 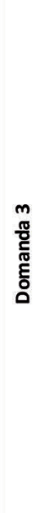 } & \multirow{7}{*}{ 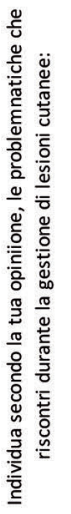 } & \multicolumn{2}{|c|}{ Poca conoscenza dell' argomento } & 20 & $34,48 \%$ & 15 & $46,88 \%$ & 5 & $23,81 \%$ & $\mathbf{0}$ & $0,00 \%$ \\
\hline & & \multicolumn{2}{|c|}{ Mancata collaborazione tra operatori } & 14 & $24,14 \%$ & 10 & $31,25 \%$ & 3 & $14,29 \%$ & 1 & $20,00 \%$ \\
\hline & & \multicolumn{2}{|c|}{ Mancata collaborazione con caregiver } & 22 & $37,93 \%$ & 12 & $37,50 \%$ & 10 & $47,62 \%$ & 0 & $0,00 \%$ \\
\hline & & \multicolumn{2}{|c|}{ Assenza di medicazioni appropriate } & 48 & $82,76 \%$ & 25 & $78,13 \%$ & 19 & $90,48 \%$ & 4 & $80,00 \%$ \\
\hline & & \multicolumn{2}{|c|}{ Assenza di materiale in generale } & 54 & $93,10 \%$ & 30 & $93,75 \%$ & 19 & $90,48 \%$ & 5 & $100,00 \%$ \\
\hline & & \multicolumn{2}{|c|}{$\begin{array}{l}\text { Accessi settimanali al pz non conformi } \\
\text { alla tipologia di medicazione }\end{array}$} & 19 & $32,76 \%$ & 11 & $34,38 \%$ & 4 & $19,05 \%$ & 4 & $80,00 \%$ \\
\hline & & \multicolumn{2}{|l|}{ Altro } & 9 & $15,52 \%$ & 8 & $25,00 \%$ & 1 & $4,76 \%$ & $\mathbf{0}$ & $0,00 \%$ \\
\hline
\end{tabular}

Figura 7. Dati questionario operatore. 
prescrivere uno schema di medicazione, ma solo nel $32,4 \%$ vengono utilizzate medicazioni avanzate $(n=107)$.

In una popolazione di studio dove si è analizzato che il $40,21 \%$ risulta a rischio di insorgenza di lesioni da pressione e il $25,54 \%$ risulta ad essere ad alto rischio di insorgenza, solo nel 9,79\% dei pazienti gli infermieri compilano ed utilizzano scale di valutazione nel corso della gestione del paziente in Assistenza Domiciliare Integrata. Valutata l'alta prevalenza delle lesioni da pressione in assistenza domiciliare forse ci si deve porre il quesito su come mai l'infermiere non compila scale di valutazione per individuare così i pazienti a rischio.

Il 79,31\% degli infermieri del territorio che hanno aderito allo studio hanno sentito parlare della figura dell'infermiere specialista in Wound Care e il 98,28\% degli operatori afferma di ritenere utile la presenza di uno specialista Wound Care in grado di coordinare i vari professionisti ed impostare adeguate strategie terapeutiche, mostrando l'esigenza di aver bisogno di una figura che li aiuti nella gestione dei pazienti con lesioni cutanee croniche.

Come analisi epidemiologica descrittiva, questo studio vuole essere una "fotografia" della distribuzione delle lesioni cutanee croniche e della loro gestione nell'assistenza domiciliare integrata nei distretti di Sassari, Alghero e Ozieri della regione Sardegna, con tutti i limiti che uno studio descrittivo comporta.

Limitazione di questo studio è stata la poca esperienza nel condurre tale tipo di analisi e la rielaborazione dei numerosi dati raccolti.

Può essere utilizzato come ipotesi di un futuro studio epidemiologico e per ottenere dati statistici sulle lesioni cutanee croniche della regione Sardegna.

\section{CONCLUSIONI}

Lo studio fa emergere l'entità del problema dei pazienti con lesioni cutanee croniche nell'assistenza domiciliare integrata nei distretti di Sassari, Alghero e Ozieri della regione Sardegna.

Si sono ottenuti, per la prima volta, dati inequivocabili che dimostrano la reale dimensione del problema, e risulta evidente la rilevanza sociale ma anche economica di questo fenomeno.

Queste conclusioni supportano la necessità di proseguire con un futuro progetto di studio che approfondisca ulteriormente la panoramica del territorio sardo nell'ambito del Wound Care per elaborare una strategia che possa favorire un'omogeneizzazione dei comportamenti dei professionisti sanitari, determinando una riduzione dei costi, ma soprattutto favorire un miglioramento della qualità di vita dei soggetti portatori di lesioni cutanee.

Si è riscontrato inoltre la necessità di uno specialista in Wound Care, il quale può collaborare attivamente con i professionisti sanitari attraverso attività di consulenza, supervisione dei processi operativi, ma può offrire anche la possibilità di creare un percorso strutturato che guidi le scelte dell'operatore che gestisce tali pazienti.

Questo lavoro perciò vuole essere un primo passo per cercare di aiutare i pazienti portatori di lesioni cutanee croniche in assistenza domiciliare integrata del territorio sardo, ma anche i professionisti sanitari che li prendono in cura approfondendo il fenomeno e le modalità di gestione.

Si auspica che, mettendo alla luce le problematiche della gestione del paziente con lesioni cutanee croniche, si possa cercare di risolvere, o almeno migliorare le problematiche riscontrate sul territorio.

\section{BIBLIOGRAFIA}

1. Gabbiani G. The myofibroblast in wound healing and fibrocontractive diseases. J Pathol 2003;200:500-3

2. Zhao G, Usui ML, Lippman SI et al. Biofilms and inflammation in Chronic Wounds. Adv Wound Care 2013;2: 389-99

3. Scalise A. Lesioni cutanee croniche gestione e trattamento. Edra: 2015: pp. 2-3.

4. Campitiello F, Lauriello C. Percorsi diagnostico-terapeutici ospedale-territorio per la gestione delle ulcere cutanee. AIUC: 2012: pp 7-8.

5. Associazione Italiana Ulcere Cutanee - onlus. Progetto S.I.UC. (Studio Italiano Ulcere Cutanee). Disponibile presso: http://www.aiuc.it/files/ProgettoSIUCWUWHS.docx.pdf 